\title{
Study of monomolecular layers of azobenzene derivative by the scanning probe microscopy
}

\author{
A.M. Alexeev*, E.A. Kosobrodova, I.V. Myagkov \\ The State Research Institute for Physical Problems, Zelenograd 103460, Russia
}

\begin{abstract}
In the paper, the study of the surface topography of a monomolecular layer of an azobenzene derivative deposited on a hydrophilic substrate as a function of temperature is proposed. At the temperature of $75{ }^{\circ} \mathrm{C}$, formation of plain needle-shape crystals was observed for $100 \mathrm{~min}$. When stored at room temperature conditions, the crystals spread back on the substrate surface. The typical time of spreading was 10 h. At further heating-cooling cycles, the pattern was repeated. A model is proposed for the explanation of experimental results.

(C) 2002 Elsevier Science B.V. All rights reserved.
\end{abstract}

Keywords: Scanning probe microscopy; Monomolecular layers; Azobenzene derivative; Growth of crystals

\section{Introduction}

The development of scanning probe microscopy offered a new opportunity for study of morphology and structure of mono- and multimolecular (Langmuir-Blodgett) films [1] as well as common organic films [2-4] on solid substrates. In particular, it enables the observation of variations induced by temperature in an object in nanometer range using multipass techniques or simultaneous study of object topography in atomic-force scanning mode and electric potential jump measurements at the surface of the object. Such combined analysis allows more thorough research into the nature of the object. Of great importance is the fact that these experiments can be carried out on a fixed predefined part of the surface of a sample.

In the present paper, the behaviour of monomolecular layer of $p$-( $p$-octadecyl-aminophenylazo) benzenesulfamide (mel-7) deposited on a smooth hydrophilic substrate is discussed. This surface-active substance has the interesting feature that in the temperature range $65-115{ }^{\circ} \mathrm{C}$, it is in smectic liquid-crystal phase, while at higher temperatures, it transfers to liquid isotropic phase. Previously [5], LB films were studied by means of small-angle X-ray diffraction. At room temperature, the LB films were found to contain twophases and have microcrystalline areas with the layer lines

\footnotetext{
* Corresponding author. Tel.: +7-95-535-0305; fax: +7-95-535-6410.

E-mail address: alexander@ntmdt.ru (A.M. Alexeev).
}

period of 47.9 and $40.1 \AA$. When heated to $70{ }^{\circ} \mathrm{C}$, only one bimolecular layer period of $37.9 \AA$ appeared. After cooling, the whole volume of the LB film had a structure with one layer period of $37.3 \AA$.

The molecule of mel-7 has high dipole moment of about 12 D [3]. Therefore, considerable electrostatic inhomogeneity was expected to be observed at the breaks of the monomolecular layer at the solid substrate. This inhomogeneity was actually present, but it appeared less pronounced, being three to five times smaller than expected. However, after heating the samples, the growth of highly anisotropic in shape of needle-like crystals was observed. The height of the crystals in the direction normal to the substrate corresponds to an odd number of monomolecular layers. It is remarkable that after $12 \mathrm{~h}$ at a room temperature, the crystals spread over the surface producing a fairly homogeneous monomolecular layer. In the paper, a possible cause is proposed for such reversible behaviour of monolayers at thermocycling.

\section{Experimental results}

In the present work, mono- and multilayers of $p$ - $(p$ octadecyl-aminophenylazo)benzenesulfamide (mel-7) were studied. The compound mel-7 was chromatographically pure; it was synthesized in the Research Institute for Organic Semiproducts and Dyes (Moscow, Russia). Solution of the compound was prepared in the mixture of the 
solvents: methanol-chloroform-benzene (volume ratio 1:1:6). The concentration was $1 \mathrm{mg} / \mathrm{ml}$.

mel-7

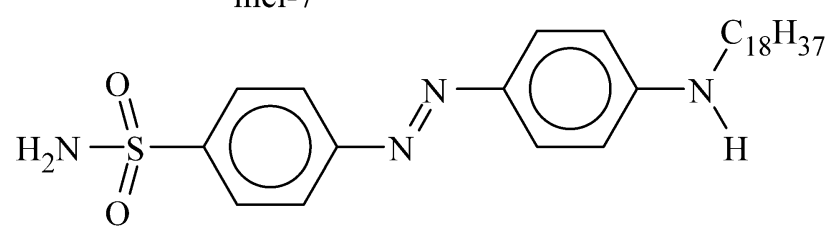

Distilled water was used as a subphase. The temperature of the water and the air was $18-20{ }^{\circ} \mathrm{C}$ with relative air humidity of $60-80 \%$.

The formation of the mono- and multilayers was made on the NT-MDT LB5 trough. The trough area is $45 \times 11 \mathrm{~cm}^{2}$. The surface pressure was measured by Wilhelmy plate made of filtering paper. The rectangular silicon (111) plates with the thickness of $0.35 \mathrm{~mm}$ were used as a substrate. Before the deposition of the monolayer, the silicon plate was loaded into oxygen plasma. After that, the substrate became hydrophilic.

The transfer of the monomolecular layer from the water surface to the substrate was performed at the surface pressure of $33 \mathrm{mN} / \mathrm{m}$. The speed of the withdrawal of the substrate from the water subphase was $20 \mathrm{~mm} / \mathrm{min}$. The transfer coefficient approached unity.

The investigation of the changing surface topography as a function of temperature was carried out by means of SPM SOLVER P47 (NT-MDT, Russia). The topography and the surface potential image were obtained simultaneously. The surface potential image was measured by the Kelvin method. The topography was obtained in tapping mode.

\section{Results and discussion}

Fig. 1 shows three histograms of surface irregularity, for the original silicon substrate (1), for monolayer of mel-7 before heating (2) and the monolayer spread over the substrate after the heating-cooling cycle (3). It is evident from the figure that histograms for (1) and (2) practically coincide. This supports that the structure of the organised monomolecular layer is fairly perfect. Fig. $2 \mathrm{a}-\mathrm{d}$ represents subsequent topography pictures from a fixed part of the sample at the temperature of $75^{\circ} \mathrm{C}$ at the initial moment (a), after $11 \mathrm{~min}$ (b), $16 \mathrm{~min}$ (c) and $127 \mathrm{~min}$ (d). The characteristic size of the studied area was $35 \times 35 \mu \mathrm{m}^{2}$. Dark aureoles around needle-shape crystallites are apparent on Fig. $2 \mathrm{~b}$ and c. The regions seem to be depleted of mel-7 molecules. The aureoles around crystallites are absent from the samples with large exposure time at $75{ }^{\circ} \mathrm{C}$ (see Fig. 2d). This corresponds to the condition when all the material of the original monolayer was accumulated in the crystallites. It should be noted that the pattern shown in Fig. $2 d$ does not vary with time, i.e. becomes stable. Quantitative analysis of
Fig. 3a revealed that the volume of the crystallites was approximately (within 10\%) equal to that of the original monolayer on the substrate. This suggests that at heating to $75{ }^{\circ} \mathrm{C}$, the evaporation of molecules of mel-7 takes place rather slowly, the energy of molecule adhesion to the substrate being sufficiently large.

The measurements of surface potential were performed in Kelvin-mode using double-pass techniques. Fig. 3 represents topography (a) and surface potential image (b) simultaneously obtained on the fixed part of the sample (50 $\mathrm{min}$ at $75{ }^{\circ} \mathrm{C}$.) The aureoles around the needle-shape crystallites (see Fig. $2 b$ and c) were also present when measuring the surface potential (Fig. 3b).

Fig. 4a shows a fragment of a microcrystallite in expanded scale (the area of $2.5 \times 2.5 \mu \mathrm{m}^{2}$ ), while the profiles of two characteristic sections $\mathrm{AA}^{\prime}$ and $\mathrm{B}^{\prime} \mathrm{B}$ are given in Fig. $4 \mathrm{~b}$ and c, correspondingly. Near the crystallite, a typical area of clean substrate surface without the monolayer is evident in Fig. 4b (segment CD).

The analysis of the profile sections $\mathrm{AA}^{\prime}$ and $\mathrm{B}^{\prime} \mathrm{B}$ (Fig. $4 \mathrm{~b}$ and c) shows that the crystals have typical steps with the thickness of $40 \pm 2 \AA$. The height of separate steps over the substrate surface satisfies the condition $d=20+40 n$, where the first item stands for the thickness of one monolayer on the substrate, 40 is the thickness of bimolecular layer in $\AA$ and $n$ is the number of bilayers in the structure. The highest with respect to crystal thickness had $n=7$. The value of the steps is in good accordance with the parameters of bimolecular period of the structure of LB film from mel-7, obtained in Ref. [5] at $70{ }^{\circ} \mathrm{C}$ or at a room temperature after annealing. Other important characteristics of the crystals of mel-7 on the substrate surface include the typical length of $10-30 \mu \mathrm{m}$ and width of $0.3-1.0 \mu \mathrm{m}$. These crystals are practically flat, their thickness being $\sim 30 \mathrm{~nm}$, which is 10-30 times less than their width.

The most remarkable result in this work was the fact of spreading of the crystal material into a homogeneous monomolecular layer after the samples were stored at room temperature. When measuring topography, it was found that

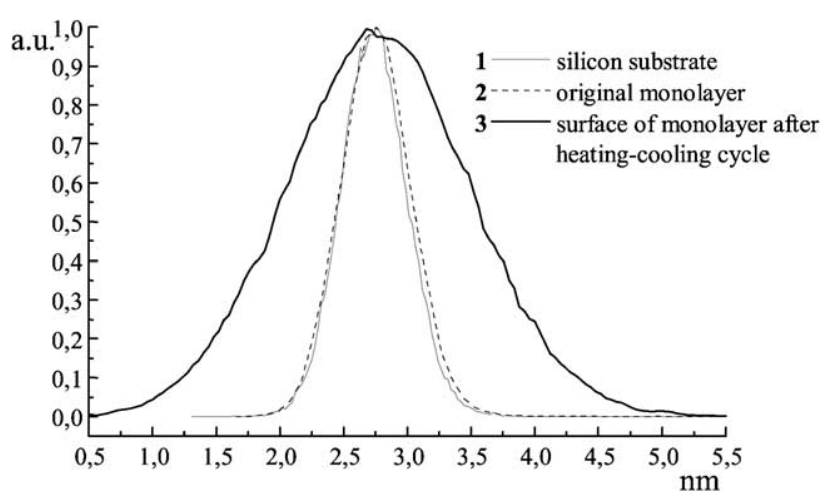

Fig. 1. Normalized frequency function of relief heights of the surface obtained using an atomic-force microscope on the levels of the original silicon substrate (1), with the deposited monomolecular layer of mel-7 (2) and with the layer after the first heating-cooling cycle in $12 \mathrm{~h}$ (3). 
a)

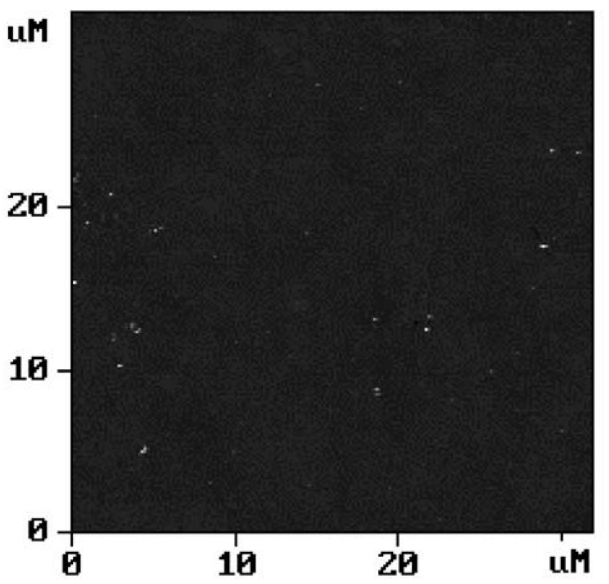

c)

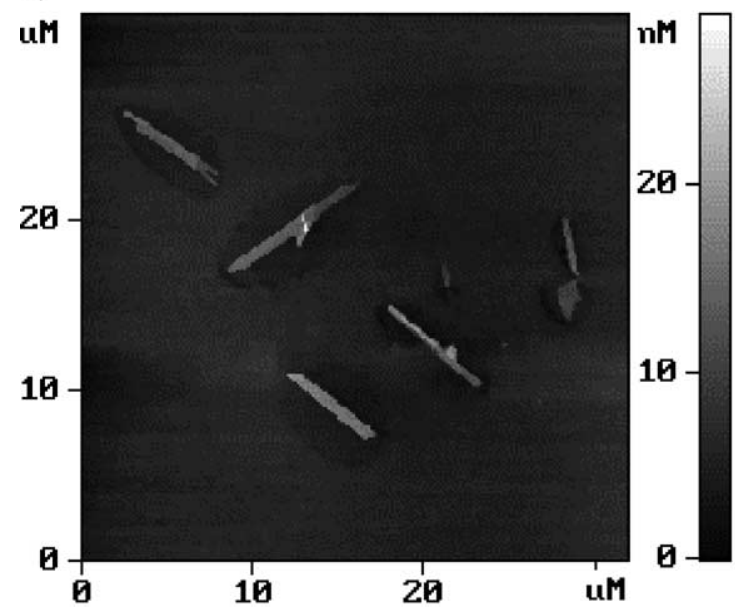

b)
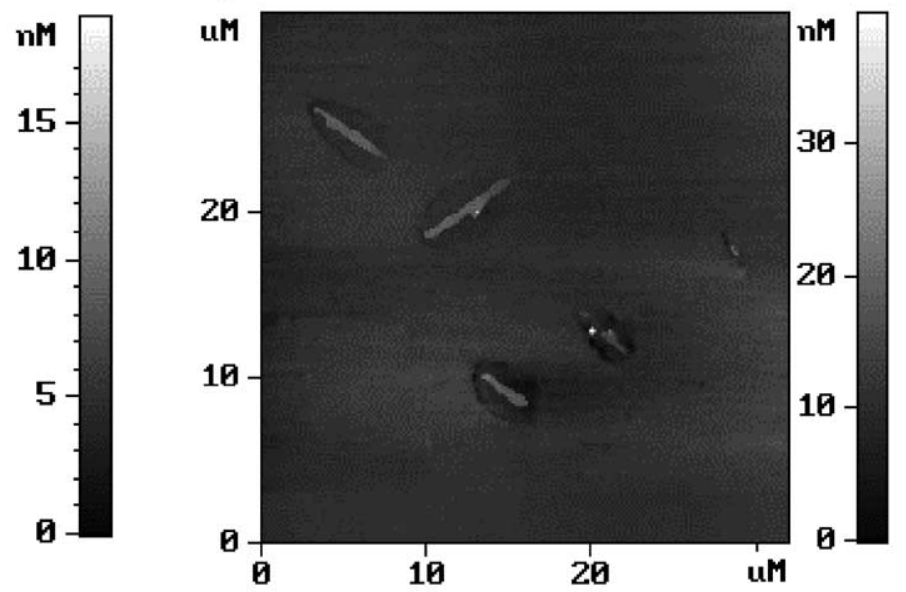

d)

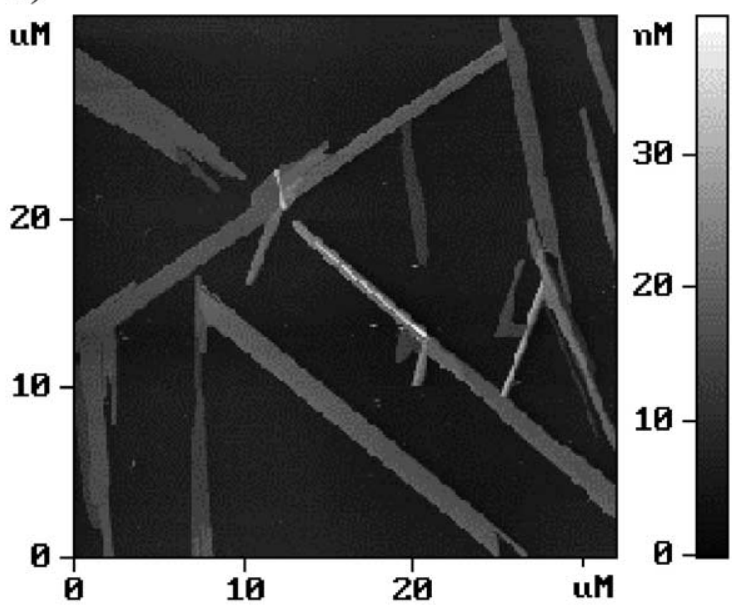

Fig. 2. Variation of surface topography of the monolayer of mel-7 on silicon substrate as a function of time at the constant temperature of $75{ }^{\circ} \mathrm{C}$ in the initial moment (a), after $11 \mathrm{~min}(\mathrm{~b}), 16 \mathrm{~min}(\mathrm{c})$ and $127 \mathrm{~min}(\mathrm{~d})$.

the crystals disappeared in $10-12 \mathrm{~h}$ in the conditions of air atmosphere. Curve 3 in Fig. 1 shows typical surface irregularities. Molecules of mel-7 have high molecular weight and did not evaporate in a noticeable extent even at a high temperature $\left(75^{\circ} \mathrm{C}\right)$ for $2 \mathrm{~h}$. Consequently, there was no doubt that the substance was left on the surface. An additional check for the presence of the monolayer on the substrate surface was made. In the contact mode on a smooth region of the substrate, test scribing of rectangular shape was made. At further analysis of this region using tapping mode, the rectangular region cleaned from the monolayer was distinctly visible on the substrate surface, having the typical step of $\sim 20 \AA$. After subsequent heating and cooling to room temperature, the pattern of crystal shaping and redistribution into monolayer repeated. Thus, one can speak about reversibility of the crystal shaping since after cooling, the crystals spread into monolayer in a characteristic time of $12 \mathrm{~h}$. Such metamorphoses of monomolecular layers on a solid substrate were not reported earlier.
In this paper, the following explanation of the observed reversible transformations of the monolayer of mel-7 was proposed. After deposition of the monomolecular layer of mel-7 on the substrate, some amount of water (apparently, on the level of one to three monolayers) is left between polar groups of the monolayer and the substrate. The water layer stabilises the organised structure of the monomolecular layer of mel-7 due to the ability of the former to increase mean energy of interaction between polar groups and the substrate, at the account of hydrogen bonds formation. At heating to $75{ }^{\circ} \mathrm{C}$, the water is removed and some of the hydrogen bonds break. Consequently, the mean energy of interaction between the molecules and the substrate decreases, and their free energy grows. In such conditions, thermodynamically, it is more advantageous for the monolayer substance to transform into crystallite form. In layered crystals of mel-7 (for instance, in LB films), the hydrogen bonds are formed at the contact of polar groups between adjacent monolayers. As a result, free energy of molecules decreases. Above the point of the phase transition of 
a)
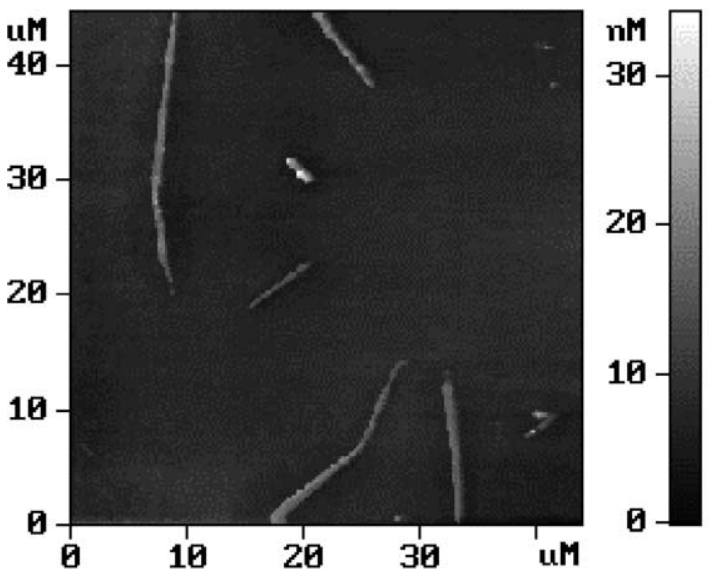

b)

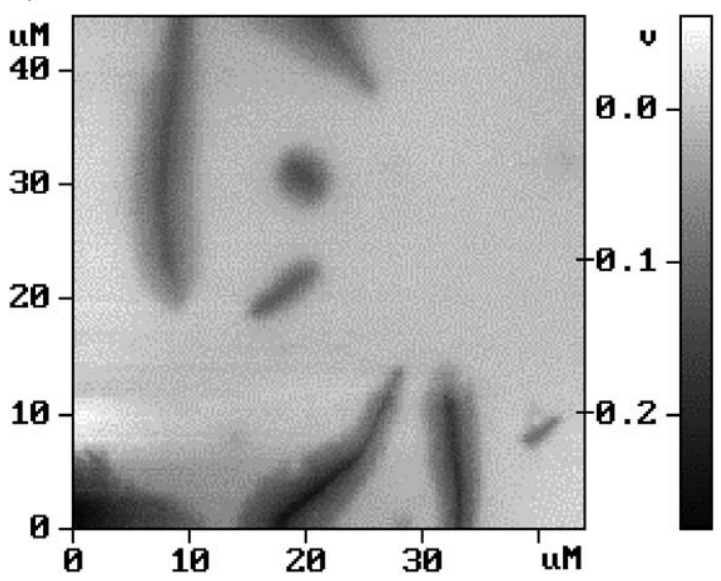

Fig. 3. (a) Topography of the substrate surface in 50 min after heating to $75^{\circ} \mathrm{C}$ and (b) electrical potential distribution over the same area.

monolayer of mel-7 into smectic state, the energy of intermolecular interaction in planar direction (cohesion energy) decreases. Therefore, the activation barrier for the transition of molecules from monolayer structure to the free substrate surface also decreases. Molecule diffusion on the substrate surface does not reduce significantly, despite the absence of water due to the rise of temperature. As a result, the growth of the crystals on the substrate surface is observed during 50-100 min. After cooling in room conditions, water from air atmosphere in small amounts is adsorbed at the surface of hydrophilic substrate. This seems enough for the substance from crystallites to start spreading into a monolayer on the substrate surface. A full analogue of this process is the behaviour of small crystals of surfaceactive substance on the water surface.

Within this interpretation of the phenomenon, it is easy to draw the energy diagram of the process of growth and disappearance of the crystals on the surface of hydrophilic substrate during heating and cooling. Fig. 5 contains a schematic diagram of the energy state of molecules of mel-7 in the crystal structure on the surface of clean substrate and in a dense monolayer. The thin line corre- sponds to heated substrate. The thick line represents the case of not heated substrate with a thin water layer between the monolayer and the substrate surface. The value of the activation barrier $E_{1}$ for crystal spreading can be obtained from the speed of transition of molecules of mel-7 from the crystals to the monolayer on the substrate surface. Naturally,

a)

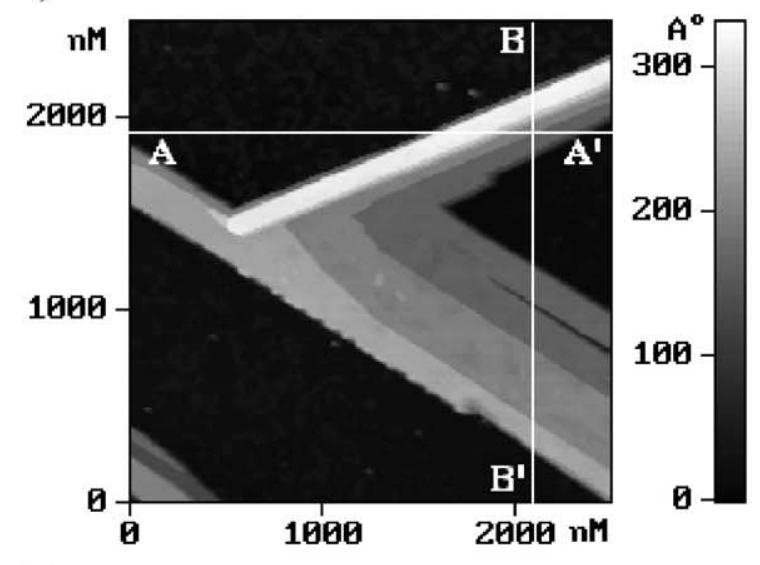

b)

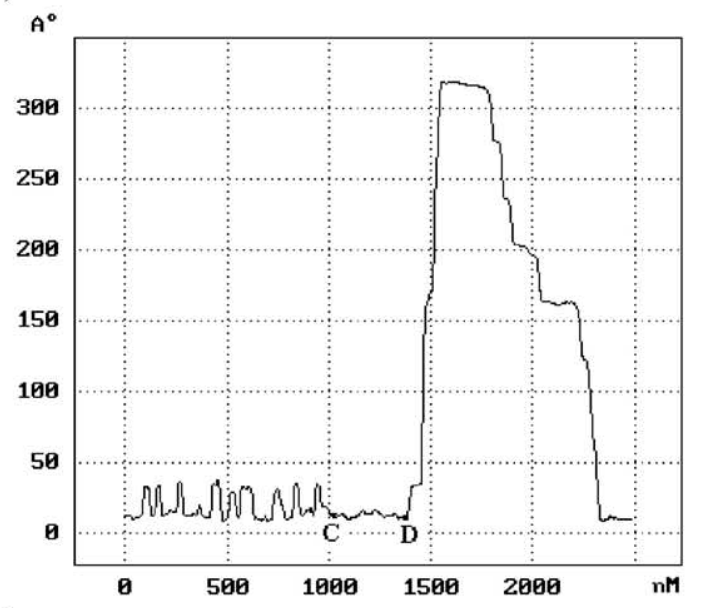

c)

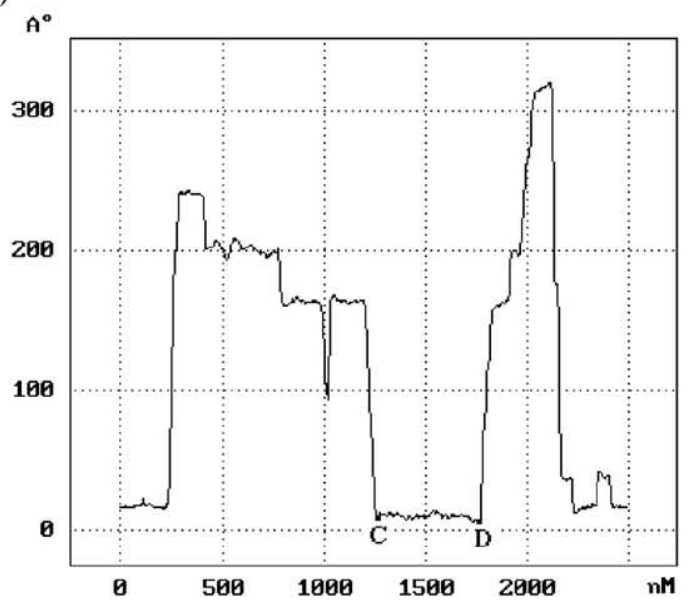

Fig. 4. (a) Layered crystals of mel-7 in expanded scale; (b) and (c) section profiles along the lines $\mathrm{AA}^{\prime}$ and $\mathrm{B}^{\prime} \mathrm{B}$, correspondingly. 


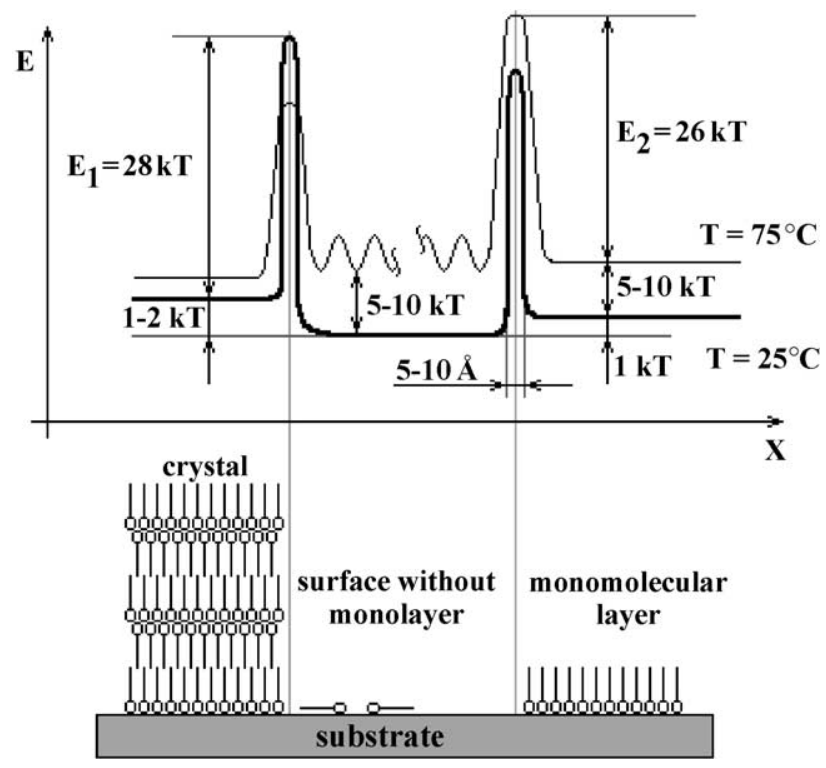

Fig. 5. Schematic diagram of the energy state of molecules of mel-7 in the crystal structure on the clear surface of the substrate and in the structure of the monomolecular layer on the surface of the hydrophilic substrate. The thick line shows the free energy of the molecules of mel-7 at room temperature and the thin line is the same at $75{ }^{\circ} \mathrm{C}$. On the horizontal axis is the coordinate along the substrate and on the vertical axis is the free energy of molecules.

this transition takes place at the perimeter of the crystals. Having the characteristic dimensions of a big crystal, the surface area of $1 \times 30 \mu \mathrm{m}$ and the height of 15 monomolecular layers as well as the average crystal dissociation time of $10 \mathrm{~h}$, one obtains the mean speed of surfactant molecule transition into the monolayer on the substrate $v_{\mathrm{a}} \sim 2 \times 10^{-8}$ $\mathrm{cm} / \mathrm{s}$. The typical cross-section of the molecule of mel-7 is assumed to be $\sim 5 \times 10^{-8} \mathrm{~cm}$. If the mean spreading speed of the monolayer from the crystal through the perimeter is $\sim 2 \times 10^{-8} \mathrm{~cm} / \mathrm{s}$, then one row of surfactant molecules leaves the structure of the crystal in the time of $\sim 2.5 \mathrm{~s}$. The value of activation barrier is given by the equation:

$E_{1}=k T \ln \tau_{1} / \tau_{0}$

where $\kappa$-Boltzmann constant, $T$-absolute temperature, $\tau_{1}$ - time required to overcome the activation barrier and $\tau_{0}$ - characteristic time of molecular oscillation $\sim 10^{-12} \mathrm{~s}$ [6]. Finally, we obtained $E_{1}=28.5 \kappa T$. This is approximately $3 / 4$ of the dispersion interaction of the molecule with lateral surrounding in the structure of the crystal or dense monolayer. Similar estimation of the activation barrier $E_{2}$ for the transition of molecules from the substrate surface to the crystal at the temperature of $75{ }^{\circ} \mathrm{C}$ gives the value $E_{2}=26.2 \kappa T$. However, this value may be an equivalent of distributed energy barrier for the separation of molecules from the monolayer and further diffuse motion from the monolayer to the crystal along the free substrate surface. At room temperature, the energy of molecules on the free substrate surface differs from that in the crystal structure (or in the dense monolayer) by nearly $1-2 \kappa T$ [7]. When the water is removed by heating, the mean free energy of molecules can increase to a value comparable with the energy of one or even two hydrogen bonds. The formation of the crystals will be energetically advantageous if this value changes just on $5-10 \kappa T$. The variation of the free energy of molecules in crystals caused by the removal of the water from the space between the first layer of molecules and the substrate is inversely proportional to the number of layers in the crystal, and thus constitutes a small value of $\sim 1 \kappa T$.

Generally, the free surface energy on the sides of the crystal is not a form-shaping factor for crystals. As a rule, the key role here is deemed to be played by temperature and composition irregularities as well as by thermodiffusion conditions of the crystallized component. In the experiments being analyzed, the temperature was equal all over the sample, the crystal dimensions being measured in microns. Actually, we are faced with two-dimensional transition of the substance by diffuse manner without thermoconvection. Therefore, from the analysis of the crystal geometry, it can be concluded that the free surface energy on the crystal ends is about $2.3 \kappa T$ larger than on its sides. In turn, the energy on the sides is larger than on its top by also $2.3 \kappa T$. The elongated shape of the crystals seems to be determined by anisotropy of the dispersion interaction in the area of rigid aromatic fragments. The interaction along the planes of azobenzene fragments is greater than that along the side edges of aromatic ones. If this is the main reason for the elongated shape of the crystals, then the planes of azobenzene fragments must be directed normally to the sides of the crystal major axis.

\section{Conclusions}

On the basis of the undertaken analysis of the phenomenon, it is possible to formulate primary conditions for the formation of the crystals from surfactants, when heating the monomolecular layer, and their subsequent spreading on the surface at a room temperature in air atmosphere:

1. The substrate should be hydrophilic.

2. The surfactant should have the phase of smectic liquidcrystal state in the temperature range above the temperature conditions of the monomolecular layer formation. This condition ensures the reformation of the monolayer into crystals in attainable time.

3 . The growth of crystals can be observed in the temperature range of the smectic state of the surfactant. The temperature should be sufficient for the water to evaporate from the substrate surface.

4. On the water subphase, at a low surface pressure, the surfactant monolayers should be in the liquid-crystal state. This condition provides spreading of the surfactant over the substrate with a thin water layer in sufficiently small observation time of $\sim 10 \mathrm{~h}$. 
5. Crystal spreading over the substrate can be observed in the air atmosphere with the relative humidity above $60 \%$.

The experiments carried out in this work prove that heating the hydrophilic substrate up to $70-75{ }^{\circ} \mathrm{C}$ is an efficient way to remove small amounts of water from its surface. This allows to avoid various artifacts during the work with atomic-force microscope which arise from the presence of water on the open hydrophilic surfaces of the substrate. Another method to remove water from the hydrophilic substrate is working in dry atmosphere.

\section{References}

[1] D.K. Schwartz, Surf. Sci. Rep. 27 (1997) 241

[2] C. Marieta, M. del Rio, I. Harismendy, I. Mondragon, Eur. Polym. J. 36 (7) $(2000) 1445$.

[3] L.G.M. Beekmans, G.J. Vancso, Polymer 41 (25) (2000) 8975.

[4] J. Kikkawa, J. Inoue, H. Abe, T. Iwata, J. Doi, Polymer 42 (6) (2001) 2707.

[5] E.A. Kosobrodova, I.V. Myagkov, V.R. Novak, V.V. Belyaev, Kolloid Z. 64 (3) (2002) 1.

[6] Ya.I. Frenkel, Kinetic Theory of Liquids, Nauka, Leningrad, 1975, p. 592.

[7] G.L. Gaines, Insoluble Monolayers at Liquid-Gas Interface, WileyInterscience, New York, 1966. 